\title{
Aurora-A is an efficient marker for predicting poor prognosis in human nasopharyngeal carcinoma with aggressive local invasion: 208 cases with a 10-year follow-up from a single institution
}

\author{
ZHI-GANG LIU ${ }^{1,2}$, WEI YI ${ }^{1,2}$, YA-LAN TAO ${ }^{1,2}$, HSIAO CHANG CHAN ${ }^{3}$, MU-SHENG ZENG $^{1}$ and YUN-FEI XIA ${ }^{1,2}$ \\ ${ }^{1}$ State Key Laboratory of Oncology in Southern China; ${ }^{2}$ Department of Radiation Oncology, Cancer Center, \\ Sun Yat-Sen University, Guangzhou 510060; ${ }^{3}$ Epithelial Cell Biology Research Center, Department of Physiology, \\ Faculty of Medicine, The Chinese University of Hong Kong, Shatin, Hong Kong, SAR, P.R. China
}

Received December 14, 2011; Accepted February 28, 2012

DOI: 10.3892/ol.2012.660

\begin{abstract}
Aurora-A kinase (Aur-A), a member of a family of mitotic serine/threonine kinases, is known to be amplified in epithelial malignancies. In this study, we focused our investigation on Aur-A expression and its prognostic significance in nasopharyngeal carcinoma (NPC). Immunohistochemical staining for Aur-A was performed on the paraffin sections of 208 patients with NPC. Data were subjected to statistical analysis with respect to clinicopathological variables, overall survival and disease-free survival. An immunohistochemical analysis showed that Aur-A was highly expressed in $132(63.5 \%)$ of the 208 NPC tissues examined. Aur-A expression was significantly correlated with $\mathrm{T}$ classification $(\mathrm{P}=0.012)$, clinical stage $(\mathrm{P}=0.003)$ and skull base invasion $(\mathrm{P}=0.003)$. Statistical analysis showed that Aur-A expression was inversely correlated with the 10 -year overall and diseasefree survival rates of NPC patients. Results of the multivariate analysis revealed that Aur-A expression was an independent prognostic indicator for patient survival. More significantly, Aur-A was found to be a marker for poor survival, which was mainly attributed to its high expression in the subgroup of $\mathrm{T}_{4}$ tumor classification with aggressive local invasion. These results indicated that Aur-A expression is inversely correlated with survival and directly correlated with the malignant status of NPC. Therefore, Aur-A may serve as a potential biological marker for poor prognosis in the $\mathrm{T}_{4}$ subgroup of patients.
\end{abstract}

\section{Introduction}

Nasopharyngeal carcinoma (NPC) has the highest incidence in Southern China and is one of the most common types of

Correspondence to: Professor Yun-Fei Xia, State Key Laboratory of Oncology in South China, Cancer Center, Sun Yat-Sen University, 651 Dongfeng Road East, Guangzhou 510060, P.R. China

E-mail: xiayf@sysucc.org.cn

Key words: nasopharyngeal carcinoma, Aurora-A, prognosis, $\mathrm{T}_{4}$ subgroup patients cancer in Southeast Asia $(1,2)$. The possible etiological factors identified for NPC are genetic susceptibility, Epstein-Barr virus (EBV) infection and environmental risk factors. Although plasma EBV DNA quantification has been recommended as an independent biomarker for the prediction of survival $(3,4)$, currently the prediction of prognosis for patients with NPC mainly depends on clinical staging. However, NPC patients with the same clinical stage often present different clinical outcomes, suggesting that the clinical staging is insufficient for accurate prediction of the prognosis of NPC.

NPC is more radiosensitive than other malignant tumors arising in the head and neck, making radiotherapy an important treatment modality for non-disseminated NPC. Although NPC is usually curable by radiotherapy, there are incidents of local failure and metastases $(5,6)$. According to the clinical and biological behavior of NPC patients following radiotherapy, we subdivided NPC into four types: Type I (no primary and regional recurrence and no distant metastasis), type II (primary or regional recurrence and no distant metastasis), type III (no primary and regional recurrence, and distant metastasis) and type IV (primary or regional recurrence, and distant metastasis) (7). The identification of prognostic factors that are more accurately correlated with treatment outcome would help in determining which NPC patients may benefit from a higher radiation dose or should undergo combined treatment with more aggressive chemotherapy. A number of candidate biological markers, such as p53, EGFR and Bcl-2 genes, have been reported. However, none of these markers have been established as a marker in decision-making for the treatment of NPC. Therefore, establishment of a biological marker that may determine prognosis and response to a particular treatment is essential in improving prognosis and developing individualized strategies, thus enhancing treatment outcome.

Aurora-A kinase (Aur-A), a member of the serine/threonine kinase family, is required for centrosome maturation and separation, mitotic commitment, chromosome alignment and possibly cytokinesis $(8,9)$. Aur-A is located on chromosome 20q13.2, which is frequently amplified in epithelial cancers $(10,11)$, suggesting the possible link of its dysregulation to carcinogenesis. Aur-A has been indicated as an oncogene since the ectopic overexpression of Aur-A in NIH3T3 and 
Rat-1 has been shown to induce cell transformation that generates tumors when implanted in nude mice (11-13). In previous clinical studies, the Aur-A gene was found to be amplified or upregulated in various types of cancer characterized by chromosome instability (CIN), which is in agreement with the previous experimental results suggesting that Aur-A abnormalities are involved in carcinogenesis (14-18).

While a positive correlation of Aur-A abnormalities with aggressive tumor behavior and poor prognosis has been reported in recent studies with clinical tissues of certain cancers $(14,16,19-21)$, a poor correlation has also been reported for other types of cancer, indicating a possible dependence of the correlation on specific types of cancer (22-25). Previous studies indicated that Aur-A downregulation was capable of inducing cell death, reducing migration and enhancing radiosensitivity in human NPC. The epithelial-mesenchymal transition (EMT) is an important process that mediates cancer invasion and metastasis, and links to radioresistance $(26,27)$. Additionally, Aur-A inhibition attenuated EMT (28). Since the correlation between Aur-A status and disease progression has not been fully investigated in NPC, the present study was conducted to assess the expression of Aur-A in NPC and its correlation to the clinicopathological parameters, overall survival and diseasefree survival in 208 NPC patients.

\section{Materials and methods}

Tissue samples. This study was conducted on a total of 208 paraffin-embedded NPC samples obtained from patients who were histologically and clinically diagnosed at the Sun Yat-Sen University Cancer Center, China, between 1994 and 1999. Patient consent and approval from the Institute Research Ethics Committee was obtained prior to the use of these clinical materials for research purposes. The clinical characteristics of the patients are shown in Table I. There were 156 males and 52 females, with a median age of 48.1 years (range, 13-72).

The routine staging workup included a detailed clinical examination of the head and neck, fiberoptic nasopharyngoscopy, computed tomography (CT) imaging of the entire neck from the base of the skull, chest radiography, abdominal sonography, a complete blood count and a biochemical profile. The disease stages of all 208 patients were classified or reclassified according to the 1992 NPC staging system of China as mentioned in a previous study (29). Follow-up information was obtained from all 208 patients. In total, 162 patients were treated with radiotherapy, six patients with chemotherapy, and 40 patients received both treatments. Radiotherapy was administered as 2 Gy daily fractions, 5 days per week, for a total intended dose of $70 \mathrm{~Gy}$. Additional (boost) radiotherapy of 8-12 Gy was delivered for residual tumor and destructed skull base following a standard dose of $70 \mathrm{~Gy}$. The neck received 50-70 Gy depending on the lymph node involvement, 50 Gy for node-negative necks and 60-70 Gy for node-positive necks. At 3 months following the completion of radiotherapy, the radiotherapy response was evaluated by clinical examination, CT scan and biopsy when required.

Immunohistochemical staining (IHC). IHC staining was performed using Dako Envision system (Dako, Carpinteria, CA, USA) according to the manufacturer's instructions. Briefly,
Table I. Clinicopathological characteristics of patient samples and expression of Aurora-A in NPC.

\begin{tabular}{|c|c|}
\hline Characteristics & $\mathrm{N}(\%)$ \\
\hline \multicolumn{2}{|l|}{ Gender } \\
\hline Male & $156(75.0)$ \\
\hline Female & $52(25.0)$ \\
\hline \multicolumn{2}{|l|}{ Age (y) } \\
\hline$\leq 45$ & $97(46.6)$ \\
\hline$>45$ & $111(53.4)$ \\
\hline \multicolumn{2}{|c|}{ Histological classification (WHO) } \\
\hline Type II & $10(4.8)$ \\
\hline Type III & $198(95.2)$ \\
\hline \multicolumn{2}{|c|}{ Clinical stage (92 stage) } \\
\hline I & $26(12.5)$ \\
\hline II & $64(30.8)$ \\
\hline III & $53(25.5)$ \\
\hline IV & $65(31.3)$ \\
\hline
\end{tabular}

T classification

T1

$93(44.7)$

$\mathrm{T} 2$

$40(19.2)$

$40(19.2)$

T4

$35(16.8)$

$\mathrm{N}$ classification

N0

$128(61.5)$

N1

$33(15.9)$

$\mathrm{N} 2$

$30(14.4)$

N3

$17(8.2)$

Distant metastasis

Yes

$29(13.9)$

No

$179(86.1)$

Vital status (at follow-up)

Alive

$136(65.4)$

Mortality due to NPC

72 (34.6)

Therapy

Radiation therapy alone

$162(77.9)$

Chemotherapy alone

Radiation therapy + chemotherapy

40 (19.2)

Skull base invasion

Yes

42 (20.2)

No

$166(79.8)$

Relapse

Yes

23 (11.1)

No

185 (88.9)

Expression of Aurora-A

Low

$76(36.5)$

High

$132(63.5)$

NPC, nasopharyngeal carcinoma

paraffin sections $(4 \mu \mathrm{m})$ were baked for $1 \mathrm{~h}$ at $65^{\circ} \mathrm{C}$. The sections were deparaffinized in xylenes and rehydrated with graded ethanol to distilled water. The sections were submerged 
into EDTA antigenic retrieval buffer $(\mathrm{pH} 8.0)$ and microwaved for antigenic retrieval. After being treated with $0.3 \% \mathrm{H}_{2} \mathrm{O}_{2}$ for 15 min to block the endogenous peroxidase, the sections were treated with normal goat serum for $30 \mathrm{~min}$ to reduce the non-specific binding, and then rabbit polyclonal anti-AurA antibody (1:300; Upstate Biotechnology, Charlottesville, VA, USA) was incubated with the sections overnight at $4^{\circ} \mathrm{C}$. After washing, the sections were incubated with horseradish peroxidase (HRP) at $4^{\circ} \mathrm{C}$ for $30 \mathrm{~min}$. Diaminobenzidine (DAB) was used for the color reaction. For the negative controls, the antibody was replaced with normal goat serum.

Evaluation of staining. The immunohistochemically stained tissue sections were scored separately by two pathologists blinded to the clinical parameters. For Aur-A assessment, the entire tissue section was scanned to assign the scores. The staining intensity was scored as 0 (negative), 1 (weak), 2 (medium) or 3 (strong). The extent of staining was scored as $0(0 \%), 1(1-25 \%), 2$ (26-50\%), $3(51-75 \%)$ and $4(76-100 \%)$, according to the percentages of the positive staining areas in relation to the whole carcinoma area, or entire section for the normal samples. The sum of the intensity and extent score was used as the final staining scores (0 to 7) for Aur-A. This relatively simple, reproducible scoring method that yields highly concordant results between independent evaluators has been used in previous studies $(30,31)$. An optimal cut-off value was selected on the basis of a measure of heterogeneity with the log-rank test with respect to overall survival. For the purpose of statistical evaluation, tumors having a final staining score of $\leq 3$ were classified as tumors with a low expression and those with a score of $>3$ were classified as tumors with a high expression of Aur-A antigen.

Statistical analysis. Statistical analysis was carried out using the SPSS 10.0 statistical software package. The Mann-Whitney $\mathrm{U}$ test was used to analyze the Aur-A expression and clinicopathological characteristics. Survival curves were plotted by the Kaplan-Meier method and compared by the log-rank test. Univariate and multivariate regression analyses were performed with the Cox proportional hazards regression model to analyze the independent factors related to prognosis. $\mathrm{P}<0.05$ in all cases was considered to indicate a statistically significant difference.

\section{Results}

Clinical outcome. On completion of radiotherapy, 179 (86.1\%) of the 208 patients had an initial complete clinical response. All 208 patients had follow-up records and the median follow-up time was 61.7 months (range, 0.93-143). The overall and disease-free survival rates for all 208 patients were 65.1 and $58.7 \%$ at the end of the follow-up date, respectively, and $42(20.2 \%)$ patients had a skull base invasion. During follow-up, a total of 29 (13.9\%) patients experienced distant metastasis and $23(11.1 \%)$ patients had a relapse.

Elevated Aur-A expression in NPC tissues. In this study, we examined Aur-A expression in 208 paraffin-embedded NPC samples. Of the 208 NPC samples, 24 samples contained normal, adjacent and uninvolved nasopharyngeal columnar
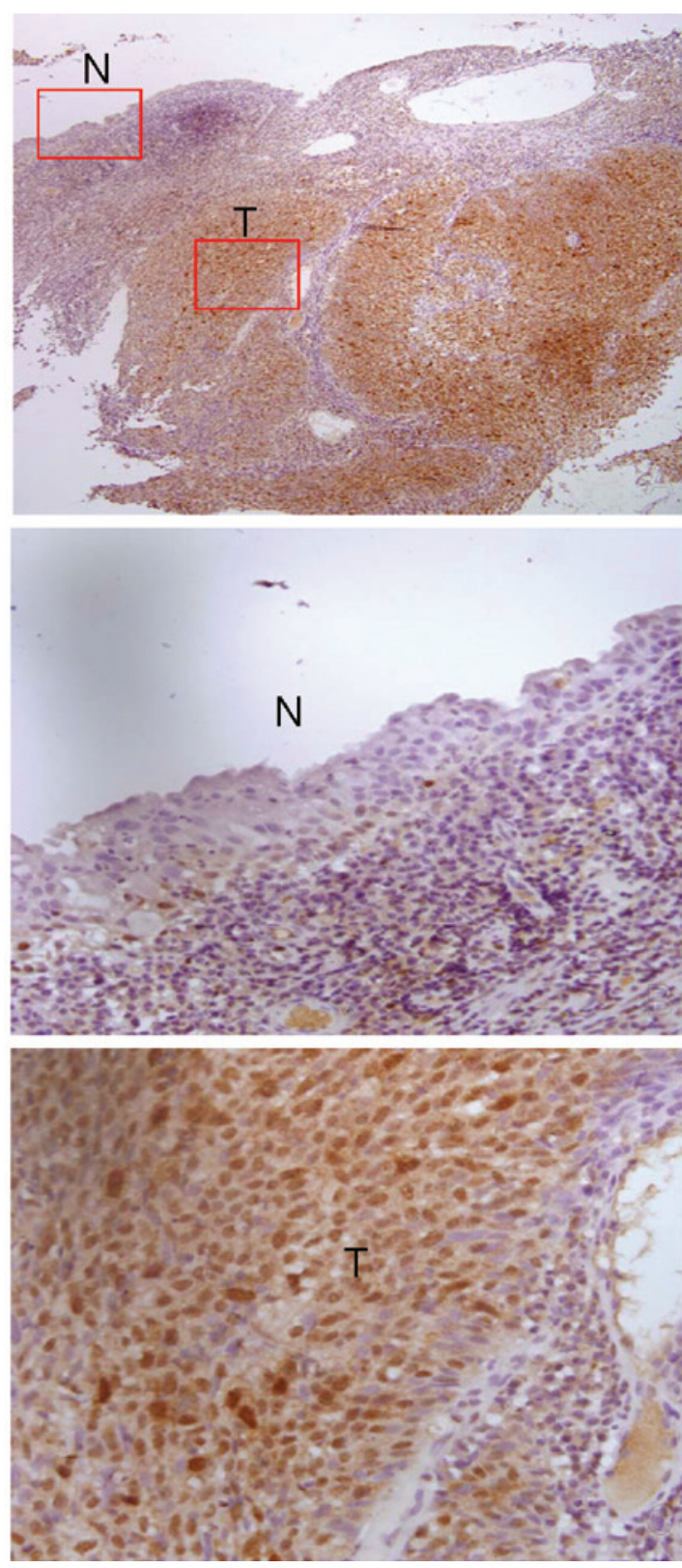

Figure 1. Increased Aur-A expression in human NPC clinical samples. NPC samples were collected and subjected to immunohistochemical staining with Aur-A antibody (DAB staining and hematoxylin counterstaining). (A) Aur-A expression in NPC and normal adjacent tissues ( $\mathrm{T}$, tumor; $\mathrm{N}$, normal) (original magnification, x200). (B) Aur-A expression in normal adjacent tissues was weak (original magnification, x400). (C) Positive expression of Aur-A in NPC samples (original magnification, $x 400$ ). NPC, nasopharyngeal carcinoma; Aur-A, Aurora-A; DAB, diaminobenzidine.

epithelia, and 12 samples contained uninvolved nasopharyngeal squamous epithelia. The majority of studies reported cytoplasmic staining of Aur-A $(32,33)$, whereas other studies showed nuclear staining $(34,35)$. Our study revealed that Aur-A localized in the nucleus and cytoplasm in NPC tissues and localized only in the nucleus in normal nasopharyngeal columnar and squamous epithelia (Fig. 1). Aur-A was highly expressed in 132 (63.5\%) of the 208 NPC tissues. The Aur-A expression level was much higher in the tumor cells compared with the adjacent normal epithelial cells, indicating an increase of Aur-A in the tumor cells (Fig. 1). 
Table II. Correlation between the clinicopathologic characteristics and expression of the Aurora-A protein.

\begin{tabular}{|c|c|c|c|}
\hline \multirow[b]{2}{*}{ Characteristics } & \multicolumn{2}{|c|}{ Aurora-A (\%) } & \multirow[b]{2}{*}{ P-value } \\
\hline & $\begin{array}{c}\text { Low } \\
\text { expression }\end{array}$ & $\begin{array}{c}\text { High } \\
\text { expression }\end{array}$ & \\
\hline \multicolumn{4}{|l|}{ Gender } \\
\hline Male & $57(36.5)$ & $99(63.5)$ & 1.000 \\
\hline Female & $19(36.5)$ & $33(63.5)$ & \\
\hline \multicolumn{4}{|l|}{ Age (y) } \\
\hline$\leq 45$ & $33(34.0)$ & $64(66.0)$ & 0.802 \\
\hline$>45$ & $43(38.7)$ & $68(61.3)$ & \\
\hline \multicolumn{4}{|c|}{ Histological classification } \\
\hline Type II & $6(60.0)$ & $4(40.0)$ & 0.115 \\
\hline Type III & $70(35.4)$ & $128(64.6)$ & \\
\hline \multicolumn{4}{|l|}{ Clinical stage } \\
\hline I-II & $43(47.8)$ & $47(52.2)$ & 0.003 \\
\hline III-IV & $33(28.0)$ & $85(72.0)$ & \\
\hline \multicolumn{4}{|l|}{ T classification } \\
\hline $\mathrm{T} 1-\mathrm{T} 2$ & $57(42.9)$ & $76(57.1)$ & 0.012 \\
\hline T3-T4 & $19(25.3)$ & $56(74.7)$ & \\
\hline \multicolumn{4}{|l|}{$\mathrm{N}$ classification } \\
\hline N0 & $49(38.3)$ & $79(61.7)$ & 0.510 \\
\hline N1-N3 & $27(33.8)$ & $53(66.2)$ & \\
\hline \multicolumn{4}{|c|}{ Distant metastasis } \\
\hline Yes & $7(24.1)$ & $22(75.9)$ & 0.136 \\
\hline No & $69(38.5)$ & $110(61.5)$ & \\
\hline \multicolumn{4}{|c|}{ Skull base invasion } \\
\hline Yes & $7(16.7)$ & $35(83.3)$ & 0.003 \\
\hline No & $69(41.6)$ & $97(58.4)$ & \\
\hline \multicolumn{4}{|l|}{ Relapse } \\
\hline Yes & $5(21.7)$ & $18(78.3)$ & 0.119 \\
\hline No & $71(38.4)$ & $114(61.6)$ & \\
\hline
\end{tabular}

Aur-A expression is associated with NPC T staging. Table II shows the association between Aur-A expression and the clinicopathological parameters. No significant correlation was found between Aur-A expression and age, gender, histological classification, $\mathrm{N}$ classification, distant metastasis or tumor relapse. However, Aur-A expression was significantly correlated with $\mathrm{T}$ classification $(\mathrm{P}=0.012)$ and skull base invasion $(\mathrm{P}=0.003)$. Moreover, Aur-A expression in late-stage NPCs was significantly higher than that in early-stage NPCs $(\mathrm{P}=0.003)$.

Inverse correlation of Aur-A overexpression with patient survival. In the entire cohort, the overall survival rate of the patients with a high Aur-A expression was significantly lower when compared with that of the low Aur-A expression group ( $\mathrm{P}=0.022$, log-rank test; Fig. 2A). The disease-free survival rate of the patients with a high Aur-A expression was also significantly lower ( $\mathrm{P}=0.017$, log-rank test; Fig. 2B). The Aur-A expression level, $\mathrm{N}$ classification, $\mathrm{T}$ classification, distance metastasis, clinical stage (92 stage) and skull base invasion were also found to be significantly correlated with survival in the Kaplan-Meier analysis and log-rank test (for $\mathrm{N}$ classification, $\mathrm{P}=0.002$; for $\mathrm{T}$ classification, distance metastasis, clinical stage and skull base invasion, $\mathrm{P}<0.001)$. Results of the univariate analysis showed that histological classification, $\mathrm{T}$ classification, clinical stage and Aur-A expression were statistically significant prognostic factors. Results of the multivariate analysis of variables in NPC patients including age, histological classification, T classification, clinical stage and Aur-A expression, demonstrated that Aur-A expression was an independent prognostic factor (Table III).

Lack of correlation of Aur-A with metastasis. When NPC patients were classified into $\mathrm{N}_{3}$ (supraclavicular lymph node, diameter $>7 \mathrm{~cm}$ or fixed or skin infiltration) or $\mathrm{M}_{1}$ (presence of distant metastasis), the tumors were considered to have a high metastatic potential. In $\mathrm{N}_{3}$ or $\mathrm{M}_{1}$ patients, Aur-A expression had no correlation with clinical outcome ( $n=40$; log-rank, $\mathrm{P}=0.85$ ) and further analysis demonstrated that Aur-A expression had no correlation with lymphatic metastasis and distant metastasis.

Close correlation of Aur-A expression with $T_{4}$ subgroup NPC patients. The patients were divided into different subgroups according to $\mathrm{N}$ classification. Notably, in the $\mathrm{N}_{0}$ (no enlarged lymph node) subgroup, we found that the patients with a high Aur-A expression had a significantly shorter survival time than those with a low Aur-A expression ( $\mathrm{n}=128$; log-rank, $\mathrm{P}=0.007$; Fig. 2C). However, in the $\mathrm{N}_{1}-\mathrm{N}_{3}$ subgroups, there were no statistically significant differences between patients with low or high levels of Aur-A expression ( $\mathrm{n}=80$; log-rank, $\mathrm{P}=0.846$ ). The $\mathrm{N}_{0}$ subgroup was divided according to $\mathrm{T}$ classification and distant metastasis, and we found that the overall survival rate of patients with a high Aur-A expression was lower than that of those with a low Aur-A expression in the $\mathrm{T}_{4} \mathrm{~N}_{0} \mathrm{M}_{0}$ subgroup $(n=30 ; P=0.042 ;$ Fig. 2D). A trend towards shorter overall survival time of patients with a high Aur-A expression was revealed in the $\mathrm{T}_{1-2} \mathrm{~N}_{0} \mathrm{M}_{0}$ subgroup, but there was no statistically significant difference $(n=61 ; \log$-rank, $P=0.176)$.

Aur-A was capable of predicting the clinical outcome of patients in the $T_{4} N_{0} M_{0}$ subgroup. Thus, we aimed to verify the correlation between Aur-A expression and the clinical outcome of $\mathrm{T}_{4}$ patients. We divided all cases into $\mathrm{T}_{1-2}, \mathrm{~T}_{3}$ and $\mathrm{T}_{4}$ subgroups. As expected, in the $\mathrm{T}_{4}$ subgroups, a high Aur-A expression had a poor clinical outcome $(n=35$; log-rank, $\mathrm{P}=0.032$; Fig. 2E), whereas Aur-A expression had no correlation to clinical outcome in either $\mathrm{T}_{1-2}$ or $\mathrm{T}_{3}$ subgroups $(\mathrm{P}=0.415$ and $\mathrm{P}=0.498$ ). Thus, regardless of $\mathrm{N}$ or $\mathrm{M}$ status, a high Aur- $\mathrm{A}$ expression indicated poor prognosis in $\mathrm{T}_{4}$ patients.

Correlation of Aur-A expression with radiosensitivity. Radiotherapy is the major treatment modality for NPC. According to our radiotherapy-related definitions for NPC (NPC patients received five-year follow-up after radical radiotherapy alone), no primary and regional recurrence was defined as radiosensitive, and primary or regional recurrence was defined as radioresistant (7). The disease-free survival (no recurrence and no metastasis) rate of the patients with a high Aur-A expression was significantly lower than a low Aur-A expression ( $\mathrm{P}=0.017$, Fig. 2B). Additionally, investigators found that Aur-A downregulation enhanced radiosensitivity in 
Table III. Univariate and multivariate analyses of various prognostic variables in patients with NPC by Cox regression analysis.

\begin{tabular}{cccc}
\multicolumn{2}{c}{ Univariate analysis } & & \multicolumn{2}{c}{ Multivariate analysis } \\
\cline { 4 - 4 } P-value & HR (95 CI) & P-value & HR (95 CI)
\end{tabular}

Age

$>45$ vs. $\leq 45$

0.085

$1.018(0.998-1.038)$

0.161

$1.244(0.703-2.201)$

Histological classification

Type II vs. III

0.031

$2.516(1.089-5.815)$

0.004

3.694 (1.532-8.906)

$\mathrm{T}$ classification

T1-2 vs. T3-4

0.000

$2.310(1.852-5.400)$

0.453

$1.244(0.703-2.201)$

Clinical stage

I-II vs. III-IV

0.000

$3.162(1.453-3.672)$

0.009

$2.448(1.254-4.777)$

Expression of Aurora-A

Low vs. high

0.024

$1.815(1.083-3.043)$

0.046

$1.746(1.011-3.014)$

NPC, nasopharyngeal carcinoma. HR, hazard ratio; CI, confidence interval.
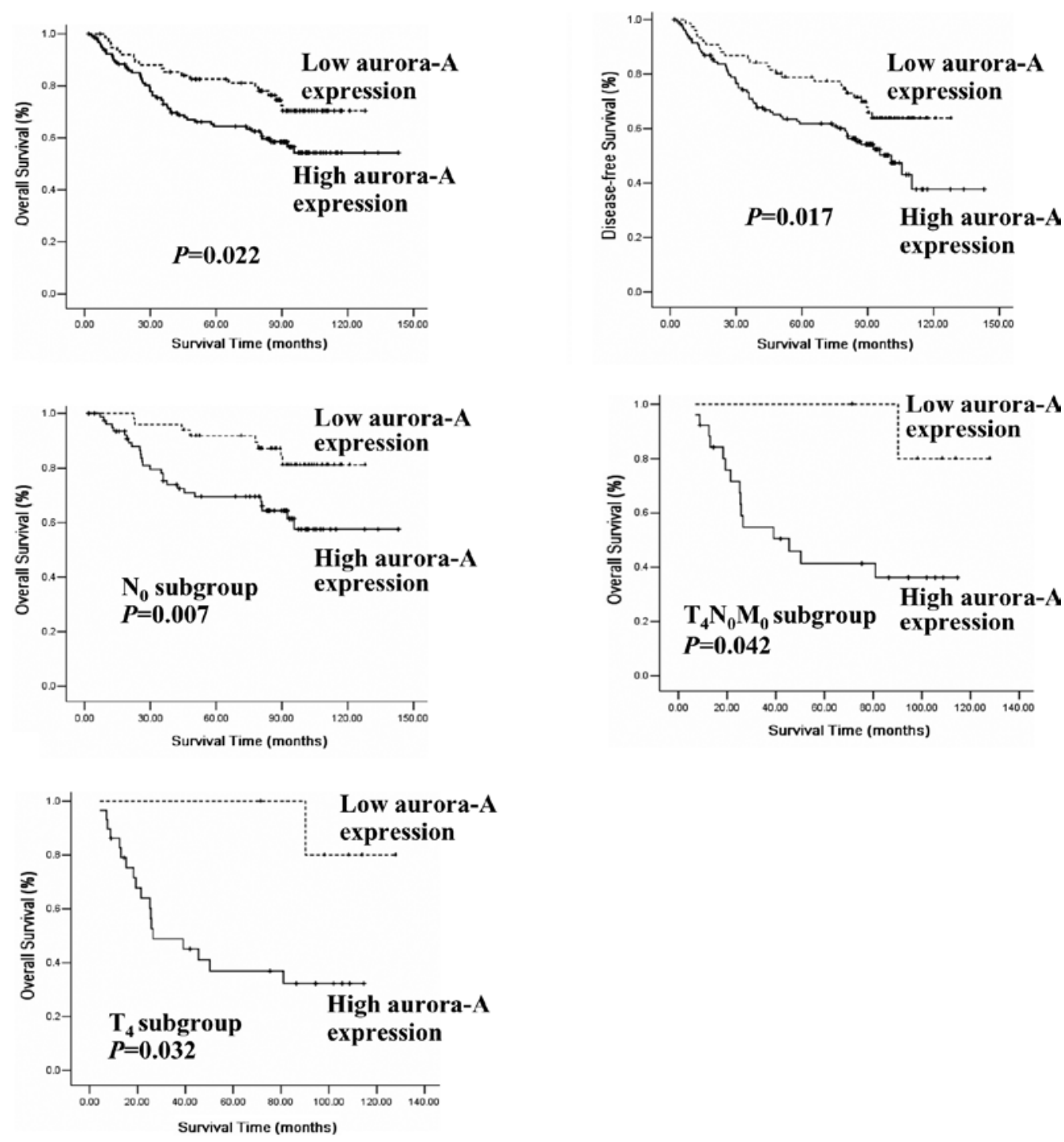

Figure 2. Kaplan-Meier analysis of survival. (A) Overall survival in all patients according to Aur-A expression. (B) Disease-free survival in all patients according to Aur-A expression. (C) Kaplan-Meier analysis of overall survival in $\mathrm{N}_{0}$ subgroup. (D) Kaplan-Meier analysis of overall survival in $\mathrm{T}_{4} \mathrm{~N}_{0} \mathrm{M}_{0}$ subgroup. (E) Kaplan-Meier analysis of overall survival in $\mathrm{T}_{4}$ subgroup. The log-rank test was used to calculate P-values. 
human NPC in vitro (36). Taken together, it indicated that a high Aur-A expression is capable of predicting radioresistance.

NPC patients with local relapse accompanied by skull base invasion have been reported to be less sensitive to radiation therapy and have a poorer clinical outcome than other patients (37). In this study, there were 7 recurrent NPC patients with skull base invasion. All 7 of these patients exhibited a high expression of Aur-A and 5 of the 7 patients (71.4\%) succumbed to NPC, indicating a possible correlation of a high Aur-A expression with radiosensitivity.

Previous studies have shown that primary tumor volume had a close correlation with survival rates and the treatment outcomes of patients with NPC $(3,18,38)$. Larger tumor volume may require more aggressive treatment, suggesting that $\mathrm{T}_{4}$ patients were not sensitive to radiation therapy. In the present study, we found that Aur-A expression served as an indicator for clinical outcome in patients of the $T_{4}$ subgroup. Therefore, radiotherapy combined with an Aur-A inhibitor may be of benefit for $\mathrm{T}_{4}$ patients.

\section{Discussion}

In this study, we have shown that Aur-A is overexpressed in NPCs compared with their normal adjacent epithelia. Our results showed that an increased expression of Aur-A was significantly associated with tumor aggressive variables, including $\mathrm{T}$ classification, skull base invasion and clinical stage. These findings are consistent with a previous report that Aur-A is overexpressed in laryngeal squamous cell carcinoma (LSCC) and associated with advanced tumor stage (16). Additionally, a significant correlation was found between a high Aur-A expression with shortened overall and disease-free survival time in NPC patients, indicating that Aur-A may be an independent prognostic factor. Consistent with our findings, certain reports have also suggested that Aur-A is a potential prognostic biomarker in breast cancer (39), bladder cancer (40), hepatocellular carcinoma (19), medulloblastoma (41), esophageal squamous cell carcinoma (35) and head and neck squamous cell carcinoma (42). However, there is controversy regarding the use of Aur-A expression as a progressive marker in glioma (23), pancreatic carcinoma (22), ovarian cancer (24) or as a prognostic marker in breast cancer (25). We speculated that the differences in the reported clinical studies may be attributed to the variability in population size, tumor characteristics, antibodies and IHC staining protocol. Our findings were obtained by analysis of a relatively large number of cases in a single institution, and thus, should be of considerable interest.

The precise mechanism by which the upregulation of Aur-A affects the process of carcinogenesis and tumor progression has yet to be clearly elucidated. A number of studies have reported that deregulation of the Aur-A function impairs genomic integrity and thus contributes to tumorigenesis by its association or interaction with its effectors or activators such as p53, TPX2, BRCA1, TACC and RASSF1A $(43,44)$. Aur-A may phosphorylate p53 at Ser215 or Ser315 and result in inactivation of the p53 transcriptional activity or facilitation of MDM2-mediated degradation $(45,46)$. Aur-A also induces telomerase activity by upregulation of c-Myc (47). Phosphorylated Aur-A may be correlated with tumor cell proliferation, inhibition of apoptosis and resistance to chemotherapeutic drugs (48). Disturbance of these factors by abnormal Aur-A expression or activity may affect tumorigenesis and tumor progression. Notably, results of previous studies revealed that a high Aur-A expression is associated with radioresistance and EMT in human NPC $(28,36)$.

A number of useful biological markers have already been identified to predict prognosis and aid in clinical decisionmaking in NPC in the following regards: high Met protein and syndecan-1 expression levels correlate with poorer survival in late-stage NPC $(49,50)$; cyclin D1 predicts NPC recurrence and tumor response to radiation therapy (51); quantification of plasma EBV DNA correlates with the response to treatment and the likelihood of relapse and survival (52); and other biomarkers such as Bmi-1, CENP-H and cathepsin D could predict the poor clinical outcome of NPC patients (53-55). Although these biological markers are likely to improve the validity of molecular classification in the future, none are capable of predicting the $\mathrm{T}_{4}$ subgroup and no small molecule inhibitor has been developed thus far.

The present data have demonstrated a statistically significant correlation between Aur-A and T classification of NPC. Particularly, in the $T_{4}$ subgroup, and even in the $T_{4} N_{0} M_{0}$ subgroup, patients with a high Aur-A expression had a poorer prognosis than those with a low Aur-A expression. Thus, we speculated that $T_{4}$ subgroup patients would benefit from the chemical inhibition of Aurora kinase to improve treatment outcome. Recently, Aurora kinase inhibitors have been developed and VX-680 has shown promising results in in vivo studies (44). The potential of sensitizing NPC cells to radiotherapy by inhibiting Aur-A activity provided the feasibility of combinational treatment for the $\mathrm{T}_{4}$ NPC patient subgroup.

The correlation analysis in the present study does not appear to indicate a significant correlation of Aur-A with lymphatic or distant metastasis, in contrast to a previous report on head and neck squamous cell carcinoma (HNSCC) showing that the upregulation of Aur-A was significantly associated with tumor distant metastasis (42). Furthermore, the present study has shown that in $\mathrm{N}_{3}$ or $\mathrm{M}_{1}$ subgroup patients, Aur-A expression does not appear to have a correlation with clinical outcome, indicating that Aur-A activity is not linked to metastasis and that Aur-A may not be involved in the metastatic process, at least in NPC. The difference of Aur-A expression in NPC and HNSCC may be attributed to the unique features of NPC. NPC is distinctive in head and neck carcinoma for its marked geographical variation shown in incidence, its close association with $\mathrm{EBV}$, its highly metastatic nature and its unique clinical development features $(56,57)$.

Taken together, results of the present study have demonstrated that the level of Aur-A expression was highly elevated in NPC, inversely correlated with survival and directly correlated with the malignant status of NPC. The present findings suggests that Aur-A is a biological marker that may be used to identify a subgroup of patients with poor prognosis for new treatment approaches. An Aurora kinase inhibitor that sensitizes NPC cells to radiotherapy would offer an opportunity for future target-guided combinational therapy.

\section{Acknowledgements}

We thank Professor Quentin Liu for his valuable comments and extensive editing of the manuscript. This study was supported 
by grants from the National High Technology Research and Development Program of China (No. 2006AA02Z4B4) and the National Natural Science Foundation of China (No. 30770641 and 31170805).

\section{References}

1. Licitra L, Bernier J, Cvitkovic E, et al: Cancer of the nasopharynx. Crit Rev Oncol Hematol 45: 199-213, 2003.

2. Titcomb CP Jr: High incidence of nasopharyngeal carcinoma in Asia. J Insur Med 33: 235-238, 2001.

3. Chen MK, Chen TH, Liu JP, Chang CC and Chie WC: Better prediction of prognosis for patients with nasopharyngeal carcinoma using primary tumor volume. Cancer 100: 2160-2166, 2004.

4. Leung SF, Zee B, Ma BB, et al: Plasma Epstein-Barr viral deoxyribonucleic acid quantitation complements tumor-nodemetastasis staging prognostication in nasopharyngeal carcinoma. J Clin Oncol 24: 5414-5418, 2006.

5. Sham JS and Choy D: Prognostic factors of nasopharyngeal carcinoma: a review of 759 patients. Br J Radiol 63: 51-58, 1990.

6. Lee AW, Poon YF, Foo W, et al: Retrospective analysis of 5037 patients with nasopharyngeal carcinoma treated during 19761985: overall survival and patterns of failure. Int J Radiat Oncol Biol Phys 23: 261-270, 1992.

7. Li ZQ, Xia YF, Liu Q, et al: Radiotherapy-related typing in 842 patients in canton with nasopharyngeal carcinoma. Int J Radiat Oncol Biol Phys 66: 1011-1016, 2006.

8. Bolanos-Garcia VM: Aurora kinases. Int J Biochem Cell Biol 37: 1572-1577, 2005

9. Andrews PD, Knatko E, Moore WJ and Swedlow JR: Mitotic mechanics: the auroras come into view. Curr Opin Cell Biol 15: 672-683, 2003

10. Sen S, Zhou H and White RA: A putative serine/threonine kinase encoding gene BTAK on chromosome $20 \mathrm{q} 13$ is amplified and overexpressed in human breast cancer cell lines. Oncogene 14: 2195-2200, 1997.

11. Bischoff JR, Anderson L, Zhu Y, et al: A homologue of Drosophila aurora kinase is oncogenic and amplified in human colorectal cancers. EMBO J 17: 3052-3065, 1998.

12. Zhou H, Kuang J, Zhong L, et al: Tumour amplified kinase STK15/BTAK induces centrosome amplification, aneuploidy and transformation. Nat Genet 20: 189-193, 1998.

13. Giet R, Petretti C and Prigent C: Aurora kinases, aneuploidy and cancer, a coincidence or a real link? Trends Cell Biol 15: 241-250, 2005 .

14. Ogawa E, Takenaka K, Katakura H, et al: Perimembrane Aurora-A expression is a significant prognostic factor in correlation with proliferative activity in non-small-cell lung cancer (NSCLC). Ann Surg Oncol 15: 547-554, 2008.

15. Lentini L, Amato A, Schillaci T and Di Leonardo A: Simultaneous Aurora-A/STK15 overexpression and centrosome amplification induce chromosomal instability in tumour cells with a MIN phenotype. BMC Cancer 7: 212, 2007.

16. Guan Z, Wang XR, Zhu XF, et al: Aurora-A, a negative prognostic marker, increases migration and decreases radiosensitivity in cancer cells. Cancer Res 67: 10436-10444, 2007.

17. Landen CN Jr, Lin YG, Immaneni A, et al: Overexpression of the centrosomal protein Aurora-A kinase is associated with poor prognosis in epithelial ovarian cancer patients. Clin Cancer Res 13: 4098-4104, 2007.

18. Lee CC, Ho HC, Lee MS, et al: Primary tumor volume of nasopharyngeal carcinoma: significance for survival. Auris Nasus Larynx 35: 376-380, 2008.

19. Jeng YM, Peng SY, Lin CY and Hsu HC: Overexpression and amplification of Aurora-A in hepatocellular carcinoma. Clin Cancer Res 10: 2065-2071, 2004.

20. Xia LP, Zhou FF, Yang MT and Liu Q: Roles of Aurora-A in tumorigenesis and prognosis of breast cancer. Ai Zheng 28: 668-672, 2009

21. Zou LJ, Li GQ, Gong LL, et al: Expression of aurora-A kinase in human lung cancer cell lines PG, A549, and NCI-H460. Ai Zheng 24: 792-795, 2005.

22. Li D, Zhu J, Firozi PF, et al: Overexpression of oncogenic STK15/ BTAK/Aurora A kinase in human pancreatic cancer. Clin Cancer Res 9: 991-997, 2003.
23. Klein A, Reichardt W, Jung V,Zang KD, Meese E and Urbschat S: Overexpression and amplification of STK15 in human gliomas. Int J Oncol 25: 1789-1794, 2004.

24. Gritsko TM, Coppola D, Paciga JE, et al: Activation and overexpression of centrosome kinase BTAK/Aurora-A in human ovarian cancer. Clin Cancer Res 9: 1420-1426, 2003.

25. Royce ME, Xia W, Sahin AA, et al: STK15/Aurora-A expression in primary breast tumors is correlated with nuclear grade but not with prognosis. Cancer 100: 12-19, 2004.

26. Kurrey NK, Jalgaonkar SP, Joglekar AV, et al: Snail and slug mediate radioresistance and chemoresistance by antagonizing p53-mediated apoptosis and acquiring a stem-like phenotype in ovarian cancer cells. Stem Cells 27: 2059-2068, 2009.

27. Tsuji T, Ibaragi S and Hu GF: Epithelial-mesenchymal transition and cell cooperativity in metastasis. Cancer Res 69: 7135-7139, 2009.

28. Wan XB, Long ZJ, Yan M, et al: Inhibition of Aurora-A suppresses epithelial-mesenchymal transition and invasion by downregulating MAPK in nasopharyngeal carcinoma cells. Carcinogenesis 29: 1930-1937, 2008.

29. Hong MH, Mai HQ, Min HQ, Ma J, Zhang EP and Cui NJ: A comparison of the Chinese 1992 and fifth-edition International Union Against Cancer staging systems for staging nasopharyngeal carcinoma. Cancer 89: 242-247, 2000.

30. Soumaoro LT, Uetake H, Higuchi T, Takagi Y, Enomoto M and Sugihara K: Cyclooxygenase-2 expression: a significant prognostic indicator for patients with colorectal cancer. Clin Cancer Res 10: 8465-8471, 2004

31. Masunaga R, Kohno H, Dhar DK, et al: Cyclooxygenase-2 expression correlates with tumor neovascularization and prognosis in human colorectal carcinoma patients. Clin Cancer Res 6: 4064-4068, 2000.

32. Tong T, Zhong Y, Kong J, et al: Overexpression of Aurora-A contributes to malignant development of human esophageal squamous cell carcinoma. Clin Cancer Res 10: 7304-7310, 2004.

33. Hoque A, Carter J, Xia W, et al: Loss of aurora A/STK15/BTAK overexpression correlates with transition of in situ to invasive ductal carcinoma of the breast. Cancer Epidemiol Biomarkers Prev 12: 1518-1522, 2003.

34. Burum-Auensen E, De Angelis PM, Schjolberg AR, Kravik KL, Aure M and Clausen OP: Subcellular localization of the spindle proteins Aurora A, Mad2, and BUBR1 assessed by immunohistochemistry. J Histochem Cytochem 55: 477-486, 2007.

35. Tanaka E, Hashimoto Y, Ito T, et al: The clinical significance of Aurora-A/STK15/BTAK expression in human esophageal squamous cell carcinoma. Clin Cancer Res 11: 1827-1834, 2005.

36. Wan XB, Fan XJ, Chen MY, et al: Inhibition of Aurora-A results in increased cell death in 3-dimensional culture microenvironment, reduced migration and is associated with enhanced radiosensitivity in human nasopharyngeal carcinoma. Cancer Biol Ther 8: 1500-1506, 2009.

37. Farias TP, Dias FL, Lima RA, et al: Prognostic factors and outcome for nasopharyngeal carcinoma. Arch Otolaryngol Head Neck Surg 129: 794-799, 2003.

38. Chu ST, Wu PH, Chou P and Lee CC: Primary tumor volume of nasopharyngeal carcinoma: prognostic significance for recurrence and survival rate. Eur Arch Otorhinolaryngol 265 (Suppl 1): S115-S120, 2008.

39. Miyoshi Y, Iwao K, Egawa C and Noguchi S: Association of centrosomal kinase STK15/BTAK mRNA expression with chromosomal instability in human breast cancers. Int J Cancer 92: 370-373, 2001

40. Sen S, Zhou H, Zhang RD, et al: Amplification/overexpression of a mitotic kinase gene in human bladder cancer. J Natl Cancer Inst 94: 1320-1329, 2002.

41. Neben K, Korshunov A, Benner A, et al: Microarray-based screening for molecular markers in medulloblastoma revealed STK15 as independent predictor for survival. Cancer Res 64: 3103-3111, 2004

42. Reiter R, Gais P, Jutting U, et al: Aurora kinase A messenger RNA overexpression is correlated with tumor progression and shortened survival in head and neck squamous cell carcinoma. Clin Cancer Res 12: 5136-5141, 2006.

43. Marumoto T, Zhang D and Saya H: Aurora-A - a guardian of poles. Nat Rev Cancer 5: 42-50, 2005.

44. Agnese V, Bazan V, Fiorentino FP, et al: The role of Aurora-A inhibitors in cancer therapy. Ann Oncol 18 (Suppl 6): vi47-vi52, 2007. 
45. Liu Q, Kaneko S, Yang L, et al: Aurora-A abrogation of p53 DNA binding and transactivation activity by phosphorylation of serine 215. J Biol Chem 279: 52175-52182, 2004.

46. Katayama H, Sasai K, Kawai H, et al: Phosphorylation by aurora kinase A induces Mdm2-mediated destabilization and inhibition of p53. Nat Genet 36: 55-62, 2004.

47. Yang H, Ou CC, Feldman RI, Nicosia SV, Kruk PA and Cheng JQ: Aurora-A kinase regulates telomerase activity through c-Myc in human ovarian and breast epithelial cells. Cancer Res 64: 463-467, 2004.

48. Tanaka E, Hashimoto Y, Ito T, et al: The suppression of aurora-A/STK15/BTAK expression enhances chemosensitivity to docetaxel in human esophageal squamous cell carcinoma. Clin Cancer Res 13: 1331-1340, 2007.

49. Qian CN, Guo X, Cao B, et al: Met protein expression level correlates with survival in patients with late-stage nasopharyngeal carcinoma. Cancer Res 62: 589-596, 2002.

50. Chen CL and Ou DL: Expression of syndecan-1 (CD138) in nasopharyngeal carcinoma is correlated with advanced stage and poor prognosis. Hum Pathol 37: 1279-1285, 2006.

51. Lai JP, Tong CL, Hong C, et al: Association between high initial tissue levels of cyclin d1 and recurrence of nasopharyngeal carcinoma. Laryngoscope 112: 402-408, 2002.
52. Lin JC, Wang WY, Chen KY, et al: Quantification of plasma Epstein-Barr virus DNA in patients with advanced nasopharyngeal carcinoma. N Engl J Med 350: 2461-2470, 2004.

53. Song LB, Zeng MS, Liao WT, et al: Bmi-1 is a novel molecular marker of nasopharyngeal carcinoma progression and immortalizes primary human nasopharyngeal epithelial cells. Cancer Res 66: 6225-6232, 2006.

54. Cheng AL, Huang WG, Chen ZC, et al: Identificating cathepsin $\mathrm{D}$ as a biomarker for differentiation and prognosis of nasopharyngeal carcinoma by laser capture microdissection and proteomic analysis. J Proteome Res 7: 2415-2426, 2008.

55. Liao WT, Song LB, Zhang HZ, et al: Centromere protein $\mathrm{H}$ is a novel prognostic marker for nasopharyngeal carcinoma progression and overall patient survival. Clin Cancer Res 13: 508-514, 2007.

56. Chang KP, Hsu CL, Chang YL, et al: Complementary serum test of antibodies to Epstein-Barr virus nuclear antigen-1 and early antigen: a possible alternative for primary screening of nasopharyngeal carcinoma. Oral Oncol 44: 784-792, 2008.

57. Chin D, Boyle GM, Porceddu S, Theile DR, Parsons PG and Coman WB: Head and neck cancer: past, present and future. Expert Rev Anticancer Ther 6: 1111-1118, 2006. 